\title{
Road tunnel air pollution induces bronchoalveolar inflammation in healthy subjects
}

\author{
B-M. Larsson*,\#,§, M. Sehlstedtt, ${ }^{\top, \S}$, J. Grunewald ${ }^{+}$, C.M. Sköld ${ }^{+}$, A. Lundin\#, \\ A. Blomberg ", T. Sandström ${ }^{\top}$, A. Eklund ${ }^{+}$and M. Svartengren*,\#
}

ABSTRACT: Traffic-related air pollution is associated with adverse respiratory effects. The aim of the present study was to investigate whether exposure to air pollution in a road tunnel causes airway inflammatory and blood coagulation responses.

A total of 16 healthy subjects underwent bronchoscopy with bronchial mucosal biopsies and bronchoalveolar lavage (BAL) on two occasions, in random order: once at $14 \mathrm{~h}$ after a 2-h exposure to air pollution in a busy road tunnel, and once after a control day with subjects exposed to urban air during normal activities. Peripheral blood was sampled prior to bronchoscopy.

The road tunnel exposures included particulate matter with a $50 \%$ cut-off aerodynamic diameter of $2.5 \mu \mathrm{m}$, particulate matter with a $50 \%$ cut-off aerodynamic diameter of $10 \mu \mathrm{m}$ and nitrogen dioxide which had median concentrations of 64,176 and $230 \mu \mathrm{g} \cdot \mathrm{m}^{-3}$, respectively. Significantly higher numbers of BAL fluid total cell number, lymphocytes and alveolar macrophages were present after road tunnel exposure versus control. Significantly higher nuclear expression of the transcription factor component c-Jun was found in the bronchial epithelium after exposure. No upregulation of adhesion molecules or cellular infiltration was present and blood coagulation factors were unaffected.

In conclusion, exposure of healthy subjects to traffic-related air pollution resulted in a lower airway inflammatory response with cell migration, together with signs of an initiated signal transduction in the bronchial epithelium.

KEYWORDS: Air pollution, airway inflammation, bronchoalveolar lavage, cell count, particles

$\mathbf{A}$ mbient city air pollution has been shown to cause adverse health effects, including respiratory and mucosal symptoms, worsening of asthma and chronic obstructive pulmonary disease but also increased mortality due to cardiovascular disease. The major contributor to these effects has been suggested to be particulate matter (PM) pollution [1, 2]. Much of the toxicity of PM appears to be mediated by ultrafine particles produced by combustion in gasoline and diesel engines, as well as other combustion sources [3]. Additionally, road surface material, tyre- and brake-wear components and crustal matter contribute to the fine (1$2.5 \mu \mathrm{m})$ and coarse $(2.5-10 \mu \mathrm{m})$ particles, which demonstrate clear toxicological properties [4]. Particle size, chemical composition and bioavailability of chemical components have been found to be of importance in PM toxicity [5].
The airway epithelium is the primary target for environmental toxicants and substances, which can lead to inflammation and worsening of airway diseases. In exposure chamber studies, diluted diesel engine exhaust has been shown to induce airway inflammation in human subjects. This response includes activation of signal transduction pathways in epithelial cells, such as mitogen activated protein kinases, nuclear factor- $\kappa \mathrm{B}$ and activator protein (AP)-1, along with the subsequent upregulation of a range of neutrophil chemoattractant cytokines and endothelial adhesion molecule expression. This is followed by migration of neutrophils, T-cells and mast cells into the airway mucosa [6-10]. In addition to this, oxidative stress and free radicals may contribute to diesel-induced airway responses [6-9].

To date, only one study has been published that investigates health effects from exposure to road

\section{AFFILIATIONS}

*Dept of Public Health Sciences, Division of Occupational Medicine and

+Dept of Medicine, Division of Respiratory Medicine, Karolinska Institutet, and

${ }^{\text {\#} D e p t ~ o f ~ O c c u p a t i o n a l ~ a n d ~}$ Environmental Health, Stockholm Centre for Public Health, Stockholm County Council, Stockholm, and

"Dept of Respiratory Medicine and Allergy, University Hospital, Umeå, Sweden.

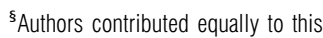
study.

CORRESPONDENCE

B-M. Larsson

Dept of Public Health Sciences Division of Occupational Medicine Karolinska Institutet

SE-171 76 Stockholm

Sweden

Fax: 468334333

E-mail: britt-marie.larsson@ki.se

Received:

March 132006

Accepted after revision:

January 072007

\section{SUPPORT STATEMENT}

The present study was supported by Karolinska Institutet (Stockholm, Sweden), the Swedish Heart and Lung Foundation (Stockholm), King Oscar II Jubilee Foundation (Stockholm) and the Swedish Environmental Protection Agency (Swedish National Air Pollution and Health Effects Program, Stockholm)

STATEMENT OF INTEREST

None declared. 
tunnel air pollution [11]. That particular traffic environment not only includes gases and PM from gasoline and diesel engines but also resuspended road dust, components from wear of running vehicles and crustal materials. In the study by SVARTENGREN et al. [11], allergic asthmatic subjects were shown to experience an increased hyperresponsiveness to inhaled allergen after a brief 30-min road tunnel exposure.

The aim of the present investigation was therefore to determine whether exposure of healthy subjects to road tunnel air pollution would result in airway inflammation, as reflected in bronchoalveolar lavage fluid (BALF) and bronchial mucosal biopsies.

\section{SUBJECTS AND METHODS Subjects}

A total of 16 healthy, nonsmoking volunteers (six female, 10 male) with a mean age of 28 yrs (range 19-59 yrs) participated in the study. All subjects underwent a physical examination, including chest radiography and blood routine examination 2 weeks before the study. None of the volunteers had a history of allergy and had not suffered airway infections within 6 weeks before exposure. Chest radiograph and lung function tests were normal and in vitro screening for the presence of specific immunoglobulin (Ig)E antibodies against common inhaled allergens (Phadiatop ${ }^{\circledR}$; Pharmacia-Upjohn, Uppsala, Sweden) was negative. No medication was allowed during the study. All participants gave their informed consent and the local ethics committee at the Karolinska Institutet (Stockholm, Sweden) approved the study.

\section{Study design}

All subjects underwent two bronchoscopies in random order, with an interval of 3-10 weeks (fig. 1). One was performed $14 \mathrm{~h}$ after a 2-h road tunnel exposure and the other (serving as a control) was performed at the same time of day and was preceded by a day of the subject's ordinary daily activities. This included work or educational activities and use of public transportation but no transit through a road tunnel. The air pollution exposure on such occasions was considered as a low urban exposure, in contrast to the road tunnel exposure.

\section{Road tunnel exposure}

The road tunnel exposures lasted for $2 \mathrm{~h}$ and took place during the afternoon rush hour (16:00-18:00 h) in the Söderleden road tunnel in the central part of Stockholm. The total length of the tunnel is $\sim 1,500 \mathrm{~m}$ and the traffic intensity is $\sim 120,000$ vehicles $\cdot$ day $^{-1}$. The subjects alternated 15-min intervals of light exercise on a bicycle ergometer $(50 \mathrm{~W})$ with 15 min of rest. Symptoms were recorded immediately before and every $20 \mathrm{~min}$ throughout the exposure.

The exposure set-up consisted of a small room $\left(\sim 6 \mathrm{~m}^{2}\right)$ located between the two tunnel tubes, some $50-100 \mathrm{~m}$ from the tunnel exit. The room had doors on either side, which were open to the adjacent traffic roads during the exposure session.

\section{Exposure measurements}

Sampling of particles with a $50 \%$ cut-off aerodynamic diameter of 2.5 and $10 \mu \mathrm{m}$ (PM2.5 and PM10, respectively) was performed using Harvard impactors (Air Diagnostics and Engineering Inc., Maine, ME, USA) at a flow rate of $10 \mathrm{~L} \cdot \mathrm{min}^{-1}$. The impactors were equipped with $37-\mathrm{mm}$ Teflon filters (Air Diagnostics and Engineering Inc.) with a pore size of $2 \mu \mathrm{m}$. Gravimetric analyses were performed after $24 \mathrm{~h}$ of conditioning using a Mettler Toledo MT5 (Mettler, Greisensee, Switzerland). The concentration of airborne ultrafine particles (aerodynamic diameter 14-100 nm) was determined using a scanning mobility particle sizer system (Electrostatic Classifier model 3071; TSI, Shoreview, MN, USA) in combination with a condensation particle counter (CPC 3010; TSI). The total number of airborne particles in the range $20-1,000 \mathrm{~nm}$ in aerodynamic diameter were monitored with a P-TRAK ${ }_{\mathbb{R}}$ particle counter (TSI). Carbon monoxide (CO) levels were measured using an electrochemical sensor (Dräger Pac III; Dräger, Luebeck, Germany). Nitric oxide (NO) levels were monitored during the exposure using a chemiluminescent instrument (AC 31M; Environnement, Poissy, France).

\section{Symptoms}

Symptoms from the eyes and upper and lower airways were recorded over the exposure session. The intensity was graded from 0 to 10, where 0 corresponded to no symptoms and 10 to severe symptoms, according to a modified Borg scale [12]. Change in symptom reporting was calculated by subtracting the pre-exposure rating from the average of all ratings during the exposure.

\section{Lung function tests}

Lung function was measured immediately before the bronchoscopies and included parameters for vital capacity, forced vital

1)

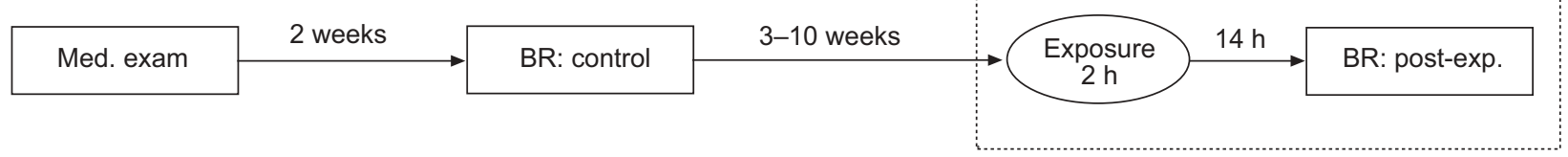

2)

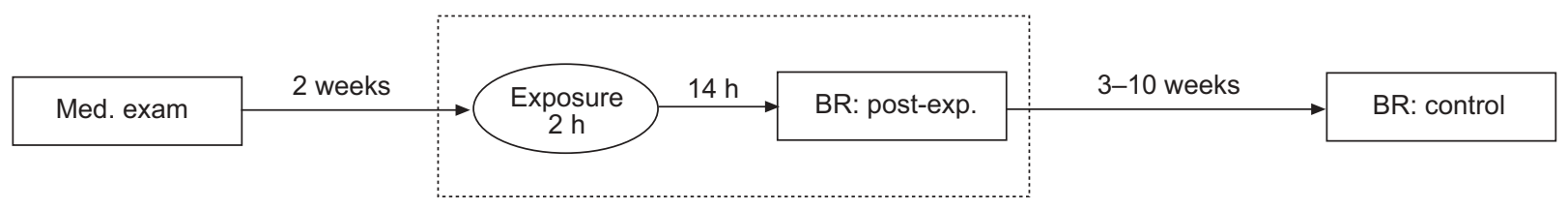

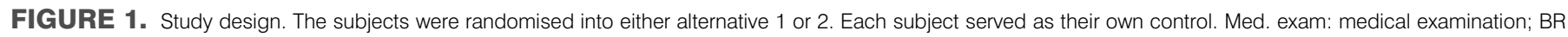
bronchoscopy; exposure 2 h: road tunnel exposure; post-exp.: post-exposure. 
capacity and forced expiratory volume in one second (FEV1), taken using a spirometer (Jaeger Masterscope; Jaeger, Würzburg, Germany).

\section{Peripheral blood}

Peripheral blood was sampled before the bronchoscopies and cell differential counts were performed using an autoanalyser (Advia 120 Hematology System; Bayer Diagnostics, Tarrytown, NY, USA). Plasminogen activator inhibitor (PAI)-1 was analysed in plasma samples using ELISA (Chromolize PAI-1; Biopool, Trinity Biotech, Bray, Ireland) and fibrinogen in plasma was analysed using a kinetic fibrinogen assay.

\section{Bronchoscopy, bronchoalveolar lavage and cell preparation}

Bronchoscopy was performed with a flexible fibreoptic bronchoscope (Olympus F Type P30; Olympus Optical Co. Ltd, Tokyo, Japan) under local anaesthesia (Lidocaine, Xylocain $_{\mathbb{R}}$; AstraZeneca, Södertälje, Sweden) and after premedication with morphine-hyoscine (Morfin-skopolamin; Meda, Solna, Sweden). Bronchial wash was performed by instilling two $10-\mathrm{mL}$ aliquots of $\mathrm{PBS}\left(37^{\circ} \mathrm{C}\right)$ in an upper lobe bronchus, and then the fluid was gently aspirated. Thereafter, five $50-\mathrm{mL}$ aliquots of PBS were instilled in the middle or lingual lobe for bronchoalveolar lavage (BAL) and the fluid was gently aspirated after each aliquot and collected in a siliconised plastic bottle kept on ice. The fluid was filtered through a single layer of Dacron net (Type AP32; Millipore, Cork, Ireland) and centrifuged at $400 \times g$ for $10 \mathrm{~min}$ at $4{ }^{\circ} \mathrm{C}$ and cells and supernatant were separated. The cells were resuspended in RPMI medium and a total cell count was performed. Smears for differential counts were prepared by cyto-centrifugation (Cytospin 2; Shandon Ltd, Runcorn, UK) at $22 \times g$ for 3 min. After staining with May-Grünwald Giemsa, 500 cells were counted. The number of mast cells in 10 visual fields ( $\times 16$ magnification) was counted after staining with toluidine/haematoxylin. Bronchial mucosal biopsies were taken from proximal cristae. BAL was performed on one side and mucosal biopsies were obtained on the contra-lateral side. At the second bronchoscopy, the sites were reversed.

\section{Flow cytometry}

BAL cells were stained with monoclonal antibodies (mAbs) against alveolar macrophage (AM) adhesion molecules, costimulatory molecules and activation markers (see online supplementary material). Isotype-matched mouse Ig mAbs were used as negative controls. For each analysis, $5 \times 10^{5}$ cells were incubated with phycoerythrine (PE) conjugated antibodies (Becton Dickinson, Mountain View, CA, USA) for $30 \mathrm{~min}$, at $4^{\circ} \mathrm{C}$, in the dark, and were thereafter washed twice with PBS. For identification of BAL cell lymphocytes, $2 \times 10^{5}$ cells were stained with fluorescein isothiocyanate, PE and RPECy-5 conjugated mAbs against CD3, CD4 and CD8 (DAKO, Glostrup, Denmark). Natural killer (NK) cells and CD56+ Tcells were stained with CD3, CD45 and CD56/CD16 (Becton Dickinson). Cells were resuspended in Cellfix (Becton Dickinson) and analysed by flow cytometry (FACSCalibur; Becton Dickinson) on the same day. Lymphocytes were gated by forward and side scatter properties, and $10^{4}$ cells were collected within the lymphocyte gate. The percentages of CD4+ and CD8+ T-cell subsets were analysed and the ratio was calculated. NK cells (defined as CD56+/CD16+/CD3-) and CD56+ T-cells (defined as CD56+/CD16+/CD3+ cells) were calculated as the percentage of all cells in the lymphocyte gate.

\section{Soluble components in BALF}

Fibronectin was analysed with a double-sandwich ELISA technique, as previously described [13]. Total matrix metalloproteinase (MMP)-9 levels (active MMP-9 and pro-MMP-9) were measured in BAL using a commercially available ELISA (R\&D Systems, Minneapolis, MN, USA).

\section{Bronchial mucosal biopsies}

The staining and quantification procedures have previously been described in detail [7, 8, 14]. Briefly, endobronchial mucosal biopsy samples were fixed overnight in a solution containing chilled acetone and protease inhibitors, before being processed into glycol methacrylate resins [15]. The fixed biopsies were cut into $2 \mu \mathrm{m}$-thin slices and placed on glass slides. Endogenous peroxidases were inhibited and nonspecific antibody binding was blocked before primary mAbs were applied and incubated overnight. mAbs were directed against early signal transduction pathways (JNK, p38, c-Jun, p65), cytokine expression (interleukin (IL)-10, IL-8, growth-related oncogene- $\alpha$ ), endothelial cells, endothelial adhesion molecule expression (p-selectin, intercellular adhesion molecule (ICAM)-1) and inflammatory cell infiltration (neutrophils, mast cells, T-cells; see online supplementary material). Finally, a biotinylated secondary antibody was added before the sections were developed and counterstained. In areas of intact epithelium and submucosa, positive staining was analysed with the assistance of computerised image analysis and light microscopy (Leica Q500IW; Leica, Cambridge, UK), as previously described $[6,14,16]$.

\section{Statistical analysis}

To analyse the responses to different exposures, the Wilcoxon's non-parametric rank sum test was used for comparisons within the same individual. The $\mathrm{p}$-values from the two-sided tests were considered significant if they were $<0.05$. Comparison of pre- and post-exposure lung function data were performed by a paired t-test. The Pearson correlation test was used in order to determine significant correlation between exposure parameters.

\section{RESULTS}

\section{Road tunnel exposure}

The median (range) PM2.5 and PM10 levels during the exposure occasions in the road tunnel throughout the afternoon rush hour were $64(46-81) \mu \mathrm{g} \cdot \mathrm{m}^{-3}$ and $176(130-206)$ $\mu \mathrm{g} \cdot \mathrm{m}^{-3}$, respectively. The median $\mathrm{NO}$ and nitrogen dioxide $\left(\mathrm{NO}_{2}\right)$ levels, respectively, were $874(751-1,032) \mu \mathrm{g} \cdot \mathrm{m}^{-3}$ and 230 $(180-269) \mu \mathrm{g} \cdot \mathrm{m}^{-3}$, and the CO level was $5.8(1.2-7.0) \mu \mathrm{g} \cdot \mathrm{m}^{-3}$. The median concentration of ambient airborne particles with an aerodynamic diameter between 20 and 1,000 nm was 1.1

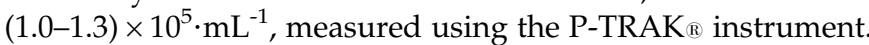
The total concentration of ultrafine airborne particles $\left(<100 \mathrm{~nm}\right.$ in diameter) was $0.85(0.74-1.0) \times 10^{5} \cdot \mathrm{mL}^{-1}$.

The $\mathrm{NO}, \mathrm{NO}_{2}, \mathrm{CO}$, ultrafine particles $(<100 \mathrm{~nm}), \mathrm{PM} 2.5$ and PM10 concentrations correlated as expected, mainly due to engine combustion origin $(p<0.01$ to $p=0.05, r=0.50$ to $\mathrm{r}=0.74)$. 


\begin{tabular}{|c|c|c|c|}
\hline TABLE 1 & $\begin{array}{l}\text { nnchoalveolar } \\
\text { d after exposi }\end{array}$ & $\begin{array}{l}\text { vage cell num } \\
\text { to road tunn }\end{array}$ & at contro \\
\hline Cells $\times 10^{6} \cdot \mathrm{L}^{-1}$ & Control & $\begin{array}{l}\text { Road tunnel } \\
\text { exposure }\end{array}$ & $p$-value \\
\hline Total cells & 80 (61-96) & $106(88-133)$ & $<0.01$ \\
\hline Lymphocytes & $7.6(5.1-11)$ & $13(9.6-13)$ & $<0.01$ \\
\hline $\begin{array}{l}\text { Alveolar } \\
\text { macrophages }\end{array}$ & 67 (53-85) & $93(62-103)$ & $<0.05$ \\
\hline Neutrophils & $1.2(0.76-1.7)$ & $1.3(0.66-2.0)$ & NS \\
\hline Eosinophils & $0.04(0-0.43)$ & $0.06(0-0.34)$ & NS \\
\hline Mast cells" & $3.5(0.5-6.8)$ & $3(1.2-6.8)$ & NS \\
\hline
\end{tabular}

The 24-h average background levels of $\mathrm{NO}_{2}, \mathrm{PM} 2.5$ and PM10 measured at a busy street level in the centre of Stockholm during the study period were 46,13 and $30 \mu \mathrm{g} \cdot \mathrm{m}^{-3}$, respectively (The City of Stockholm Environment and Health Administration, Stockholm, Sweden).

\section{Cells in BALF}

The total cell concentration in the BALF increased significantly $(p<0.01)$ after the exposure to road tunnel air versus control day with normal activity. This was mainly due to increased concentrations of lymphocytes $(\mathrm{p}<0.01)$ but also of AM $(\mathrm{p}<0.05$; table 1$)$. The percentage of lymphocytes increased significantly from 9.4 to $17 \%(\mathrm{p}<0.05)$, with a reciprocal decrease of AM from 89 to $82 \%$ after exposure $(p<0.05)$. No statistically significant changes were seen for neutrophils, eosinophils, basophils or mast cells.

\section{Flowcytometric analysis of BALF}

Analysis of lymphocyte subsets revealed a small but statistically significant decrease after exposure in the percentage of BALF NK cells $(p<0.05$; table 2$)$. In addition, BALF CD56+ Tcells decreased significantly after exposure $(p<0.05)$.

\section{Soluble components in BALF}

Fibronectin concentrations at control were 50 (interquartile range $40-129) \mathrm{ng} \cdot \mathrm{mL}^{-1}$ and were $71(41-156) \mathrm{ng} \cdot \mathrm{mL}^{-1}$ after tunnel exposure. MMP-9 concentrations were $0.54(0.23-0.83)$ $\mathrm{ng} \cdot \mathrm{mL}^{-1}$ at control and $0.32(0.22-1.03) \mathrm{ng} \cdot \mathrm{mL}^{-1}$ after tunnel exposure, without any significant difference.

\section{Bronchial mucosal biopsies}

The following stepwise immunohistochemical analyses were carried out on the biopsy material based on the expected time course of the development of inflammation in the bronchial airway mucosa, as derived from earlier investigations $[7,8,16$, 17]. 1) Early signal transduction pathways. If positive: 2) cytokine and endothelial adhesion molecule expressions were determined, and finally 3) inflammatory cell infiltration was performed.

The evaluation revealed a significant increase in the nuclear expression of c-Jun in the bronchial epithelium after exposure to road tunnel pollution versus control $(\mathrm{p}=0.034)$, with no

\begin{tabular}{|c|c|c|c|c|}
\hline TABLE 2 & \multicolumn{4}{|c|}{$\begin{array}{l}\text { Bronchoalveolar lavage fluid lymphocyte subset } \\
\text { data at control and after exposure in a road } \\
\text { tunnel }\end{array}$} \\
\hline Marker \% & & Control & $\begin{array}{c}\text { Road tunnel } \\
\text { exposure }\end{array}$ & p-value \\
\hline \multicolumn{2}{|l|}{ CD3 $+^{\#}$} & 89 (85-93) & $90(86-93)$ & NS \\
\hline \multicolumn{2}{|l|}{ CD4+ } & $69(59-73)$ & $69(62-74)$ & NS \\
\hline \multicolumn{2}{|l|}{$\mathrm{CD} 8+^{\S}$} & $24(21-33)$ & $26(21-31)$ & NS \\
\hline \multicolumn{2}{|c|}{ CD56+/CD16+/CD3- ${ }^{f}$} & $5.7(3.3-8.7)$ & $4.7(3.9-8.1)$ & $<0.05$ \\
\hline \multicolumn{2}{|c|}{ CD56+/CD16+/CD3+ ${ }^{\# \# ~}$} & $6.8(4.5-9.0)$ & $6.2(3.3-8.2)$ & $<0.05$ \\
\hline
\end{tabular}

change in the expression of the signal transduction markers JNK, p38 or p65 (table 3).

Since activation of an early signal transduction pathway was identified, the present authors proceeded with readings of cytokine expression, adhesion molecule expression and inflammatory cell counts but could not identify any significant changes in these parameters (see online supplementary material).

\section{Symptoms}

Symptom ratings increased significantly during the road tunnel exposure in the eyes (mean \pm SD $1.2 \pm 1.5 ; \mathrm{p}<0.01$ ), the upper airways $(1.4 \pm 1.2 ; \mathrm{p}<0.01)$ and the lower airways $(0.76 \pm 1.2 ; \mathrm{p}<0.05)$, as well as unpleasant smell $(1.8 \pm 1.7$; $\mathrm{p}<0.01)$.

\section{Lung function}

All subjects had a normal lung function at control and no significant changes occurred as a result of exposure.

\section{Peripheral blood}

Analysis of peripheral blood demonstrated that the total leukocyte numbers as well as plasma levels of fibrinogen and PAI-1 did not change during exposure.

\section{DISCUSSION}

In the current study, a 2-h exposure to air pollution was performed in a busy road tunnel, which caused airway inflammatory response with elevations of T-lymphocytes and $\mathrm{AM}$ in BAL, in comparison with a control day during which the subjects were exposed to urban air whilst performing normal activities. Additionally, an increased nuclear expression of the transcription factor component c-Jun was demonstrated in the bronchial epithelium after road tunnel exposure.

The air pollution exposure in the Stockholm road tunnel differed in several ways from the diluted diesel engine exhaust atmosphere investigated in a series of earlier studies $[5,7,8,14$, 16]. The PM2.5 concentration in the present study constituted $\sim 36 \%$ of the PM10 concentration, indicating that a large proportion of the particles belonged to the coarse fraction, presumably originating from road surface and tyre wear as well as resuspension of crustal material. In addition, exposure in the road tunnel included exhaust fumes from vehicles 


\begin{tabular}{|c|c|c|c|c|}
\hline TABLE 3 & \multicolumn{4}{|c|}{$\begin{array}{l}\text { Signal transduction markers in the bronchial epithelium of bronchial biopsies from healthy subjects, at control and after } \\
\text { exposure to road tunnel air pollution }\end{array}$} \\
\hline \multicolumn{2}{|c|}{$\begin{array}{l}\text { Signal transduction marker positive nuclear staining } \cdot \mathrm{mm}^{-2} \\
\text { epithelium }\end{array}$} & Control & Road tunnel exposure & $\mathrm{p}$-value \\
\hline \multicolumn{2}{|l|}{ JNK } & 467 (299-610) & $454(301-519)$ & NS \\
\hline \multicolumn{2}{|l|}{ p38 } & $72(34-175)$ & $124(36-480)$ & NS \\
\hline \multicolumn{2}{|l|}{ p65 } & 1955 (768-2738) & $1015(403-3130)$ & NS \\
\hline
\end{tabular}

running on different fuels, in contrast to the atmosphere in the controlled chamber. Gasoline dominates fuel use in Sweden and $\sim 10 \%$ of the Swedish road fleet consists of diesel-powered vehicles. The high levels of oxides of nitrogen and the concentration of ultrafine particles demonstrates that an important source of exposure is fuel combustion from motor vehicles.

Of potential importance when comparing the present study with the controlled diesel exposure studies $[5,7,8,14,16]$ is the fact that the concentration of particles with a $50 \%$ cut-off aerodynamic diameter of $1 \mu \mathrm{m}$ was considerably higher in the controlled diesel exhaust studies than that in the present study $\left(4 \times 10^{6}\right.$ particles $\cdot \mathrm{mL}^{-1}$ compared to $1 \times 10^{5}$ particles $\left.\cdot \mathrm{mL}^{-1}\right)$. Additional aspects when considering the inhaled and subsequently deposited particle dose are workload and ventilation rate. During the exercise periods of the 2-h exposure, the average minute ventilation increased to $\sim 10 \mathrm{~L} \cdot \mathrm{min}^{-1} \cdot \mathrm{m}^{-2}$, as based on ventilation measurements during step-wise exercise tests. This can be compared with $\sim 20 \mathrm{~L} \cdot \mathrm{min}^{-1} \cdot \mathrm{m}^{-2}$ during the discussed diesel exhaust exposure experiments. Nevertheless, the present exercise levels would be more representative of most exposure situations encountered in the city environment. Interestingly, despite the lower minute ventilation rate and substantially lower concentration of ultrafine particles generated by the traffic, clear airway inflammatory responses were identified.

The present study confirms earlier human experimental data, as well as animal data, from controlled diesel exhaust challenges, suggesting T-cells to be important in the airway inflammatory response $[7,10]$. T-lymphocytes are important regulatory cells that interact directly with macrophages via receptors. They also have the capacity to regulate inflammatory cell activity by cytokine release to either T-helper cell (Th) type 1 or Th2 inflammatory response. Diesel exhaust particles have been demonstrated to interact with T-cell function and may also be a potent Th2 adjuvant [18]. An author from the current study recently confirmed that the T-cell-dominated inflammation, induced by diesel exhaust exposure of $300 \mu \mathrm{g} \cdot \mathrm{m}^{3}$ PM10 for $1 \mathrm{~h}$, in healthy nonatopic subjects, leads to a Th2 shift in the bronchial epithelium, with enhanced production of IL-13 [16]. This cytokine, together with its companion IL-4, has the capacity to induce an allergic-type inflammation [16]. Worsening of asthma as a result of trafficrelated pollution has found additional support in a diesel exhaust exposure study in asthmatic subjects treated with inhaled corticosteroids. These subjects experienced a doubling of their bronchial hyperresponsiveness, in terms of a doubling difference in a provocative concentration of metacholine causing a $20 \%$ fall in FEV1 after diesel exposure versus air [19]. In addition, a preceding study by SVARTENGREN et al. [11], employing a short-term road tunnel exposure of allergic asthmatics resulted in enhanced airway responsiveness to allergen inhalation, which may also be linked to a state of enhanced Th2 response in traffic-induced air pollution.

In the present in vivo exposure study, the significant increase in BAL T-cells after exposure was not accompanied by a change in relation to $\mathrm{CD} 4$ or CD8 T-cells, indicating an unspecific accumulation of these cells. There was, however, a significant reduction in relative numbers of NK cells and cytotoxic CD56+ T-cells, both with important capacities to eliminate damaged or infected cells as well as to trigger the immune system by producing large quantities of key cytokines, such as interferon$\gamma$ and tumour necrosis factor (TNF)- $\alpha$. The decreased number of NK and cytotoxic T-cells may indicate either a relocalisation or suggest that these cells are consumed in response to the road dust exposure as part of a first line of defence against toxic substances.

BAL analysis demonstrated an increase in the concentration of $\mathrm{AM}$, in accordance with an earlier diesel engine exhaust exposure study investigating the late response at $18 \mathrm{~h}$ postexposure [6]. The concordant findings between road tunnel and diesel exposure highlight the importance of macrophages in the defence against traffic-related particulate air pollution. AM have phagocytic, antigen-presenting and immune-modulating capacities, important to the immune defence against particulates and microorganisms. The present authors have previously reported that the phagocytic ability of macrophages can be suppressed by ingestion of diesel particles during in vivo exposure of human subjects [6].

It is suggested that the rise in AM may have been due to increased clearance after tunnel exposure, while it remains to be demonstrated whether these cells were secretively active. Fibronectin, which can be produced by many different cell types, often has its origin in activated AM, and has previously been demonstrated to increase in BALF after PM exposure, resulting in elevated numbers of AM [7]. However, the results from the present study did not indicate an increased concentration of fibronectin after tunnel exposure. 
In the present study, bronchial mucosal biopsies were analysed in a stepwise manner to elucidate the stage at which a potential inflammatory response in the bronchial mucosa may occur. The analyses demonstrated an increase in nuclear translocation of c-Jun, which may indicate the presence of an early reactive epithelial response. Activation of the transcription factor AP-1, of which c-Jun is a subunit, may involve transcription of many downstream inflammatory cytokines and chemokines, including TNF- $\alpha$, IL-1 $\beta$, IL-6, IL-8 and RANTES (regulated on activation normal T-cell expressed and secreted) [20]. The present findings therefore indicate potential for neutrophilic and lymphocytic cell recruitment during the hours following biopsy sampling, through c-Jun-enhanced transcription of IL-8 and RANTES. Further support for the argument supporting a very early inflammatory state in the bronchial epithelium is the fact that the vascular adhesion molecule expressions of $\mathrm{p}$ selectin and ICAM-1 were not upregulated. Importantly, some earlier studies have suggested that inflammatory responses to air pollutants may develop more slowly after exposure to lower rather than higher concentrations. At $6 \mathrm{~h}$, controlled chamber exposure to diesel exhaust at PM10 levels of $300 \mu \mathrm{g} \cdot \mathrm{m}^{-3}[7,8,14]$ had already caused a fully established state of bronchial as well as bronchoalveolar inflammatory cell infiltration, together with upregulated vascular adhesion molecules, cytokine expressions and signal transduction pathways. In contrast, 6-h after exposure to diesel exhaust at PM10 levels of $100 \mu \mathrm{g} \cdot \mathrm{m}^{-3}$ [9] there was only an early state of inflammation, which developed substantially after $18 \mathrm{~h}$ [21]. Consequently, it is expected that the early state of inflammation $14 \mathrm{~h}$ after exposure may have developed further both in the bronchial and bronchoalveolar regions. Additional road tunnel exposure experiments including sampling at a later time point would be needed to more precisely determine the time course of the respiratory tract responses. The limited number of study subjects may have resulted in false-negative results in some of the study parameters. Ideally, a large number of subjects should have been included, but exposure studies incorporating bronchoscopies and subsequent analyses of lung samples are very resource-demanding. The current standard, based on a large number of studies, suggests a similar number of subjects as used in this study, to be sufficient to demonstrate relevant inflammatory events induced by air pollution [7-9, 13, 14, 16].

The present study addressed the hypothesis of SEATON et al. [22] that exposure to ambient air pollution would impact on the coagulation system. In the present study, no significant change in systemic coagulation as a result of road tunnel exposure was detected, a result similar to that in a recent investigation performed on human subjects following diesel exhaust exposure [23]. In contrast, the present investigators recently demonstrated that diesel exhaust may locally reduce tissue plasminogen activator release from the endothelium in vivo together with reduced vasomotor response in healthy human subjects investigated with bilateral arm plethysmography $6 \mathrm{~h}$ after exposure [23].

In conclusion, road tunnel exposure during light exercise results in airway inflammation with cell migration, reflected in bronchoalveolar lavage, together with indications of an initiated signal transduction in the bronchial epithelium as well as increased symptoms in eyes and the upper and lower airways. Forthcoming research may determine whether further inflammatory development occurs in the subsequent hours beyond the sample time point in the present study.

\section{ACKNOWLEDGEMENTS}

The authors wish to thank H. Blomqvist, M. Dahl, G. De Forest, B. Dahlberg, C. Müller-Suur and J. Pourazar for their excellent technical assistance.

\section{REFERENCES}

1 Brunekreef B, Holgate ST. Air pollution and health. Lancet 2002; 360: 1233-1242.

2 Bernstein JA, Alexis N, Barnes C, et al. Health effects of air pollution. J Allergy Clin Immunol 2004; 114: 1116-1123.

3 Donaldson K, Stone V, Clouter A, Renwick L, MacNee W. Ultrafine particles. Occup Environ Med 2001; 58: 211-216.

4 Sandstrom T, Cassee FR, Salonen R, Dybing E. Recent outcomes in European multicentre projects on ambient particulate air pollution. Toxicol Appl Pharmacol 2005; 207: Suppl. 2, 261-268.

5 Mudway IS, Stenfors N, Duggan ST, et al. An in vitro and in vivo investigation of the effects of diesel exhaust on human airway lining fluid antioxidants. Arch Biochem Biophys 2004; 423: 200-212.

6 Rudell B, Blomberg A, Helleday R, et al. Bronchoalveolar inflammation after exposure to diesel exhaust: comparison between unfiltered and particle trap filtered exhaust. Occup Environ Med 1999; 56: 527-534.

7 Salvi S, Blomberg A, Rudell B, et al. Acute inflammatory responses in the airways and peripheral blood after shortterm exposure to diesel exhaust in healthy human volunteers. Am J Respir Crit Care Med 1999; 159: 702-709.

8 Salvi SS, Nordenhall C, Blomberg A, et al. Acute exposure to diesel exhaust increases IL-8 and GRO-alpha production in healthy human airways. Am J Respir Crit Care Med 2000; 161: 550-557.

9 Stenfors N, Nordenhall C, Salvi SS, et al. Different airway inflammatory responses in asthmatic and healthy humans exposed to diesel. Eur Respir J 2004; 23: 82-86.

10 Fujimaki $\mathrm{H}, \mathrm{Ui} \mathrm{N}$, Endo T. Induction of inflammatory response of mice exposed to diesel exhaust is modulated by CD4+ and CD8+ T cells. Am J Respir Crit Care Med 2001; 164: 1867-1873.

11 Svartengren M, Strand V, Bylin G, Jarup L, Pershagen G. Short-term exposure to air pollution in a road tunnel enhances the asthmatic response to allergen. Eur Respir J 2000; 15: 716-724.

12 Rudell B, Ledin MC, Hammarstrom U, Stjernberg N, Lundback B, Sandstrom T. Effects on symptoms and lung function in humans experimentally exposed to diesel exhaust. Occup Environ Med 1996; 53: 658-662.

13 Sandstrom T, Helleday R, Bjermer L, Stjernberg N. Effects of repeated exposure to $4 \mathrm{ppm}$ nitrogen dioxide on bronchoalveolar lymphocyte subsets and macrophages in healthy men. Eur Respir J 1992; 5: 1092-1096.

14 Pourazar J, Mudway IS, Samet JM, et al. Diesel exhaust activates redox-sensitive transcription factors and kinases in human airways. Am J Physiol Lung Cell Mol Physiol 2005; 289: 724-730. 
15 Britten KM, Howarth PH, Roche WR. Immunohistochemistry on resin sections: a comparison of resin embedding techniques for small mucosal biopsies. Biotech Histochem 1993; 68: 271-280.

16 Pourazar J, Frew AJ, Blomberg A, et al. Diesel exhaust exposure enhances the expression of IL-13 in the bronchial epithelium of healthy subjects. Respir Med 2004; 98: 821-825.

17 Wilson SJ, Leone BA, Anderson D, Manning A, Holgate ST. Immunohistochemical analysis of the activation of NFkappaB and expression of associated cytokines and adhesion molecules in human models of allergic inflammation. J Pathol 1999; 189: 265-272.

18 Finkelman FD, Yang M, Orekhova T, et al. Diesel exhaust particles suppress in vivo IFN-gamma production by inhibiting cytokine effects on NK and NKT cells. J Immunol 2004; 172: 3808-3813.
19 Nordenhäll C, Pourazar J, Ledin MC, Levin JO, Sandström $\mathrm{T}$, Ädelroth E. Diesel exhaust enhances airway responsiveness in asthmatic subjects. Eur Respir J 2001; 17: 909-915.

20 Guo RF, Lentsch AB, Sarma JV, et al. Activator protein-1 activation in acute lung injury. Am J Pathol 2002; 161: 275-282.

21 Behndig AF, Mudway IS, Brown JL, et al. Airway antioxidant and inflammatory responses to diesel exhaust exposure in healthy humans. Eur Respir J 2006; 27: 359-365.

22 Seaton A, MacNee W, Donaldson K, Godden D. Particulate air pollution and acute health effects. Lancet 1995; 345: 176-178.

23 Mills NL, Tornqvist $\mathrm{H}$, Robinson SD, et al. Diesel exhaust inhalation causes vascular dysfunction and impaired endogenous fibrinolysis. Circulation 2005; 112: 3930-3936. 